\title{
Sustained enrichment of liver phospholipids and triglycerides in eicosapentaenoate after a bolus intravenous injection of a medium-chain triglycerides:fish oil emulsion to streptozotocin (Type 1) and Goto-Kakizaki (Type 2) diabetic rats
}

\author{
YVON A. CARPENTIER, DAVID FONTAINE, ANNE OTTO, \\ LAURENCE PORTOIS, JEANINE FONTAINE and WILLY J. MALAISSE \\ Laboratories of Experimental Surgery and Physiology and Pharmacology, \\ Brussels Free University, B-1070 Brussels, Belgium
}

Received November 10, 2005; Accepted January 13, 2006

\begin{abstract}
This study deals with the sustained enrichment of liver phospholipids and triglycerides in long-chain polyunsaturated $\omega 3$ fatty acids $(\omega 3)$ found after the bolus intravenous injection of a novel medium-chain triglyceride:fish oil emulsion (MCT:FO) to streptozotocin (Type 1) and GotoKakizaki (Type 2) diabetic rats. Twenty hours after injection of the MCT:FO emulsion, the relative concentration of $\omega 3$ was indeed higher in liver phospholipids and triglycerides than that found in rats injected with either saline or a control medium-chain triglyceride:long-chain triglyceride emulsion. This coincided with a decrease in the ponderal percentage of

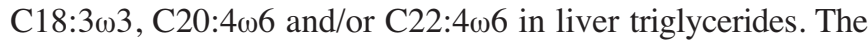
present study further documents differences between streptozotocin-induced and Goto-Kakizaki diabetic rats in terms of body weight, glycemia, liver triglyceride content and the fatty acid pattern of both liver phospholipids and triglycerides, as well as a close correlation in the latter animals between liver and plasma phospholipids or triglycerides as far as the ratio in the relative concentration of selected fatty acids representative of desaturase and elongase activities is concerned. In light of these and previous findings, it is proposed that the beneficial metabolic and functional events of the MCT:FO emulsion may display not solely a rapid but also sustained time course.
\end{abstract}

Correspondence to: Professor Willy J. Malaisse, Laboratory of Experimental Surgery, Brussels Free University, 808 Route de Lennik, B-1070 Brussels, Belgium

E-mail: malaisse@ulb.ac.be

Key words: long-chain polyunsaturated $\omega 3$ fatty acids, mediumchain triglyceride, fish oil emulsion, fatty acid content, liver phospholipids, triglycerides, streptozotocin, Goto-Kakizaki diabetic rats

\section{Introduction}

It was recently reported [Portois L, et al, Nutr Clin Metab 18 (Suppl 1): abs. S53, 2004] that the bolus intravenous injection of a medium-chain triglyceride:fish oil emulsion (MCT:FO) to normal human subjects increases within $60 \mathrm{~min}$ the content of leucocyte and platelet phospholipids in long-chain poly-

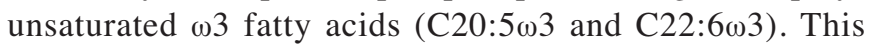
procedure was proposed, therefore, as a new approach to restore rapidly a sufficient phospholipid content of these $\omega 3$ fatty acids in selected subjects, such as those susceptible to undergo cardiac arrhythmia in response to anesthesia and surgery. Likewise, it was documented that, in both $\omega 3$-depleted rats (second generation) and normal animals examined $60 \mathrm{~min}$ after the bolus injection of the MCT:FO emulsion, a sizeable increase of the phospholipid content in $\omega 3$ fatty acids was observed in the liver, as distinct from circulating cells in direct contact with the injected lipids, this coinciding with a decrease of the hepatic triglyceride content in the $\omega 3$-depleted rats [Portois L, et al, Eur J Physiol: abs. (In press)]. In the present study, comparable experiments were conducted in animal models of either Type 1 or Type 2 diabetes, namely in rats rendered diabetic by a prior administration of streptozotocin (STZ rats) or Goto-Kakizaki rats with inherited non-insulin dependent diabetes (GK rats). The major aims of this study were to explore whether, in these diabetic rats, the injection of the MCT:FO emulsion would result in a sustained increase of the phospholipid content in $\omega 3$ fatty acids of the liver and concomitant changes in the hepatic triglyceride content and/or fatty acid composition. For the purpose of comparison other STZ and GK rats were injected with either saline or a MCT:LCT emulsion containing equal amounts (weight) of MCT lipids and soybean LCT lipids and currently used for parenteral nutrition (Medialipid ${ }^{\circledR}$, B. Braun).

\section{Materials and methods}

The MCT:FO emulsion contained 20\% (w:v) of a mixture of $80 \%$ medium-chain triacylglycerols and $20 \%$ fish oil. It also contained $2.5 \%(\mathrm{w}: \mathrm{v})$ glycerol and $1.2 \%(\mathrm{w}: \mathrm{v})$ egg-derived 
Table I. Liver phospholipid and triglyceride content and composition in STZ and GK rats injected with saline, a MCT:LCT or MCT:FO emulsion.

\begin{tabular}{|c|c|c|c|c|c|c|}
\hline \multirow{2}{*}{$\begin{array}{l}\text { Rats } \\
\text { Inoculate }\end{array}$} & \multicolumn{3}{|c|}{ STZ } & \multicolumn{3}{|c|}{ GK } \\
\hline & Saline & MCT:LCT & MCT:FO & Saline & MCT:LCT & MCT:FO \\
\hline \multicolumn{7}{|l|}{ Phospholipids } \\
\hline Total (mg/g) & $23.5 \pm 2.5(5)$ & $27.5 \pm 2.1(5)$ & $29.1 \pm 2.0(4)$ & $27.2 \pm 0.4(4)$ & $25.8 \pm 0.4(3)$ & $25.5 \pm 0.4(4)$ \\
\hline C16:0 (\%) & $17.0 \pm 0.9(5)$ & $16.1 \pm 0.5(5)$ & $16.9 \pm 0.6(4)$ & $17.2 \pm 0.7(4)$ & $16.6 \pm 1.2(3)$ & $16.5 \pm 0.5(4)$ \\
\hline 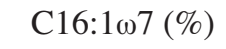 & $0.38 \pm 0.18$ & $0.46 \pm 0.16(5)$ & $0.45 \pm 0.15$ (4) & $0.50 \pm 0.07$ (4) & $0.53 \pm 0.03$ & $0.45 \pm 0.05$ \\
\hline C18:0 (\%) & $18.5 \pm 0.9(5)$ & $19.4 \pm 0.6(5)$ & $18.8 \pm 0.7$ (4) & $18.6 \pm 0.5(4)$ & $18.9 \pm 0.8(3)$ & $19.3 \pm 0.5(4)$ \\
\hline 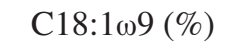 & $2.82 \pm 0.17(5)$ & $3.08 \pm 0.26(5)$ & $3.23 \pm 0.32(4)$ & $2.50 \pm 0.16(4)$ & $2.57 \pm 0.19$ & $2.23 \pm 0.15$ \\
\hline $\mathrm{C} 18: 2 \omega 6(\%)$ & $19.2 \pm 0.8(5)$ & $19.9 \pm 0.4(5)$ & $20.6 \pm 0.9$ (4) & $14.3 \pm 0.7$ (4) & $14.3 \pm 0.8(3)$ & $14.0 \pm 0.4(4)$ \\
\hline $\mathrm{C} 18: 3 \omega 6(\%)$ & $0.16 \pm 0.02(5)$ & $0.16 \pm 0.02(5)$ & $0.13 \pm 0.03$ & $0.06 \pm 0.00$ & $0.06 \pm 0.00$ & $0.05 \pm 0.00$ \\
\hline C20:4w6 (\%) & $30.7 \pm 0.9(5)$ & $30.2 \pm 0.5(5)$ & $28.5 \pm 1.4(4)$ & $32.5 \pm 0.8$ (4) & $33.2 \pm 1.2(3)$ & $33.8 \pm 0.5(4)$ \\
\hline $\mathrm{C} 20: 5 \omega 3(\%)$ & $0.28 \pm 0.09(5)$ & $0.44 \pm 0.13(5)$ & $0.55 \pm 0.10$ & $0.23 \pm 0.05$ & $0.27 \pm 0.09$ & $0.34 \pm 0.08$ \\
\hline 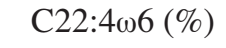 & $0.26 \pm 0.03(5)$ & $0.28 \pm 0.02(5)$ & $0.25 \pm 0.03$ & $0.43 \pm 0.03$ & $0.43 \pm 0.04$ & $0.38 \pm 0.02$ \\
\hline $\mathrm{C} 22: 5 \omega 3(\%)$ & $1.12 \pm 0.46(5)$ & $0.92 \pm 0.10(5)$ & $1.00 \pm 0.15$ (4) & $1.43 \pm 0.05(4)$ & $1.30 \pm 0.00$ & $1.38 \pm 0.06(4)$ \\
\hline $\mathrm{C} 22: 6 \omega 3(\%)$ & $6.8 \pm 0.3(5)$ & $6.0 \pm 0.5(5)$ & $6.6 \pm 0.9(4)$ & $10.1 \pm 0.4(4)$ & $9.7 \pm 0.4(3)$ & $9.5 \pm 0.3(4)$ \\
\hline \multicolumn{7}{|l|}{ Triglycerides } \\
\hline Total (mg/g) & $3.88 \pm 0.50(5)$ & $3.37 \pm 0.77$ (4) & $3.89 \pm 0.51$ (4) & $8.29 \pm 1.67$ (4) & $7.67 \pm 2.76(3)$ & $10.93 \pm 4.74(4)$ \\
\hline C16:0 (\%) & $23.1 \pm 1.3(5)$ & $22.3 \pm 1.4(5)$ & $22.3 \pm 1.7(4)$ & $24.1 \pm 1.0(4)$ & $25.2 \pm 1.1(3)$ & $24.3 \pm 0.9(4)$ \\
\hline 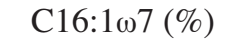 & $0.86 \pm 0.34(5)$ & $1.22 \pm 0.38(5)$ & $1.20 \pm 0.44$ (4) & $1.85 \pm 0.25(4)$ & $1.67 \pm 0.48$ & $1.98 \pm 0.09$ \\
\hline C18:0 (\%) & $2.44 \pm 0.14(5)$ & $2.48 \pm 0.35(5)$ & $2.50 \pm 0.26(4)$ & $1.88 \pm 0.09$ (4) & $2.00 \pm 0.10$ & $1.95 \pm 0.35$ \\
\hline 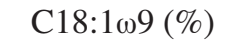 & $14.6 \pm 0.9(5)$ & $13.6 \pm 1.2(5)$ & $16.0 \pm 1.7(4)$ & $15.3 \pm 1.0(4)$ & $16.3 \pm 0.9(3)$ & $15.5 \pm 0.9(4)$ \\
\hline 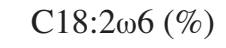 & $41.6 \pm 1.9(5)$ & $42.3 \pm 2.8(5)$ & $39.7 \pm 3.0(4)$ & $32.1 \pm 1.6(4)$ & $31.4 \pm 3.0$ & $31.8 \pm 1.5(4)$ \\
\hline C18:3w6 (\%) & $0.48 \pm 0.06(5)$ & $0.57 \pm 0.03(3)$ & $0.40 \pm 0.01$ (4) & $0.27 \pm 0.03$ & $0.26(1)$ & $0.34 \pm 0.07$ (2) \\
\hline $\mathrm{C} 18: 3 \omega 3(\%)$ & $2.34 \pm 0.12(5)$ & $2.20 \pm 0.15(5)$ & $1.68 \pm 0.14$ (4) & $1.80 \pm 0.24$ (4) & $1.70 \pm 0.17$ (3) & $1.70 \pm 0.19$ \\
\hline 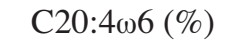 & $6.90 \pm 0.52(5)$ & $6.72 \pm 0.86(5)$ & $6.13 \pm 1.21(4)$ & $9.33 \pm 1.86(4)$ & $9.00 \pm 2.20$ & $8.10 \pm 1.21$ \\
\hline C20:5w3 (\%) & $0.86 \pm 0.13(5)$ & $0.92 \pm 0.10(5)$ & $1.03 \pm 0.24(4)$ & $0.75 \pm 0.06(4)$ & $0.73 \pm 0.07$ (3) & $1.10 \pm 0.09$ \\
\hline 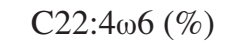 & $0.86 \pm 0.18$ & $0.86 \pm 0.17(5)$ & $0.93 \pm 0.21$ (4) & $2.20 \pm 0.28$ & $2.00 \pm 0.35$ & $1.95 \pm 0.25$ \\
\hline $\mathrm{C} 22: 5 \omega 3(\%)$ & $1.40 \pm 0.19(5)$ & $1.46 \pm 0.22(5)$ & $1.93 \pm 0.40(4)$ & $2.48 \pm 0.22(4)$ & $2.27 \pm 0.23(3)$ & $2.93 \pm 0.23$ \\
\hline C22:6w3 (\%) & $3.40 \pm 0.61(5)$ & $4.00 \pm 0.45(5)$ & $4.95 \pm 0.89$ (4) & $6.40 \pm 1.15(4)$ & $6.17 \pm 1.67$ & $7.10 \pm 1.01$ (4) \\
\hline
\end{tabular}

phospholipids as the emulsifier. The all-rac- $\alpha$-tocopherol level was $0.2 \mathrm{mg} / \mathrm{ml}$ in MCT:LCT and $4.0 \mathrm{mg} / \mathrm{ml}$ in MCT:FO.

Fed male Wistar rats (Charles River Laboratories, Brussels, Belgium) were injected intraperitoneally about five weeks before the present experiments with streptozotocin $(75 \mu \mathrm{g} / \mathrm{g}$ body weight; Sigma, St. Louis, MO, USA) freshly dissolved in saline containing a citrate buffer ( $\mathrm{pH} 4.5)$. These STZ rats and male GK rats (Taconic M\&B, Ry, Denmark) of comparable age were given free access to food (KM-04-K12; Pavan Service, Oud Turnhout, Belgium) and injected intravenously with $1.0 \mathrm{ml}$ of either saline, the MCT:FO emulsion or the MCT:LCT emulsion under chloral hydrate anesthesia. Twenty hours later, the rats were sacrificed under carbon dioxide. All animal procedures were approved by our University ethics committee.

The glycemia was measured by an Accutrend sensor (Roche Diagnostics, Vilvoorde, Belgium). The methods used to measure the content and fatty acid pattern of liver and plasma phospholipids and triglycerides were previously reported (1).

All results are expressed as mean values $( \pm$ SEM) together with the number of individual determinations (n). The statistical significance of differences between mean values was assessed by use of Student's t-test.

\section{Results}

The body weight of the animals examined in the present study averaged $324 \pm 4 \mathrm{~g}(\mathrm{n}=12)$ in GK rats, as distinct $(\mathrm{p}<0.001)$ from only $257 \pm 9 \mathrm{~g}(\mathrm{n}=14)$ in STZ rats. A reduction in body weight of STZ-injected rats was already documented on several occasions in prior studies $(2,3)$. As also expected from prior reports $(2-5)$, the glycemia was higher $(\mathrm{p}<0.01)$ in the STZ rats $(19.2 \pm 2.4 \mathrm{mM} ; \mathrm{n}=14)$ than in the GK rats $(11.1 \pm 0.8 \mathrm{mM}$; $\mathrm{n}=12)$.

Table I summarizes the results relative to the content and composition of liver phospholipids and triglycerides. Relative to hepatic wet weight, the phospholipid content of the liver, expressed as fatty acid equivalent, was not significantly different in the rats injected with saline, the MCT:FO emulsion or the other control lipid emulsion. It was also not significantly different in STZ rats $(26.5 \pm 1.4 \mathrm{mg} / \mathrm{g} ; \mathrm{n}=14)$ and GK rats $(26.2 \pm 0.3 \mathrm{mg} / \mathrm{g} ; \mathrm{n}=11)$. 
Table II. Plasma phospholipid and triglyceride concentration and composition in GK rats injected with saline, an MCT:LCT or MCT:FO emulsion

\begin{tabular}{|c|c|c|c|c|c|c|}
\hline \multirow{2}{*}{$\begin{array}{l}\text { Lipids } \\
\text { Inoculate }\end{array}$} & \multicolumn{3}{|c|}{ Phospholipids } & \multicolumn{3}{|c|}{ Triglycerides } \\
\hline & Saline & MCT:LCT & MCT:FO & Saline & MCT:LCT & MCT:FO \\
\hline Total $(\mu \mathrm{g} / \mathrm{g})$ & $660 \pm 111(4)$ & $530 \pm 102(3)$ & $637 \pm 28$ & $382 \pm 58$ (4) & $307 \pm 57$ (3) & $434 \pm 54(4)$ \\
\hline C16:0 (\%) & $22.1 \pm 1.0(4)$ & $22.4 \pm 2.1$ & $22.1 \pm 1.2(4)$ & $22.1 \pm 1.7(4)$ & $22.4 \pm 1.9$ & $22.8 \pm 1.1$ \\
\hline 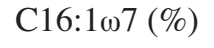 & $0.4 \pm 0.1(4)$ & $0.4 \pm 0.0(3)$ & $0.4 \pm 0.0(4)$ & $1.2 \pm 0.3(4)$ & $1.8 \pm 0.1(3)$ & $1.3 \pm 0.4(3)$ \\
\hline C18:0 (\%) & $19.6 \pm 0.7$ (4) & $20.0 \pm 1.1$ & $20.0 \pm 0.6(4)$ & $3.1 \pm 0.5(4)$ & $3.6 \pm 0.9(3)$ & $2.8 \pm 0.3(4)$ \\
\hline 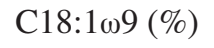 & $2.9 \pm 0.3(4)$ & $3.2 \pm 0.3(3)$ & $2.8 \pm 0.2(4)$ & $13.8 \pm 1.3(4)$ & $14.1 \pm 1.3(3)$ & $14.4 \pm 1.0$ \\
\hline 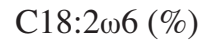 & $15.4 \pm 0.8$ & $15.9 \pm 1.0(3)$ & $16.0 \pm 0.4(4)$ & $30.7 \pm 1.9(4)$ & $30.3 \pm 3.5(3)$ & $32.8 \pm 1.8$ \\
\hline C18:3w6 (\%) & N.D. & N.D. & N.D. & N.D. & N.D. & $0.2(1)$ \\
\hline $\mathrm{C} 20: 4 \omega 6(\%)$ & $28.8 \pm 1.0(4)$ & $28.6 \pm 1.8(3)$ & $28.8 \pm 1.2(4)$ & $14.2 \pm 3.3(4)$ & $13.2 \pm 3.5$ & $10.0 \pm 1.7$ \\
\hline $\mathrm{C} 20: 5 \omega 3(\%)$ & N.D. & N.D. & N.D. & $1.3 \pm 0.1(4)$ & $1.3 \pm 0.1(3)$ & $1.6 \pm 0.1(4)$ \\
\hline $\mathrm{C} 22: 4 \omega 6(\%)$ & $0.4 \pm 0.0$ & $0.5 \pm 0.1(2)$ & $0.4 \pm 0.0$ & $1.8 \pm 0.2(4)$ & $1.5 \pm 0.3(3)$ & $1.5 \pm 0.2(4)$ \\
\hline $\mathrm{C} 22: 5 \omega 3(\%)$ & $1.1 \pm 0.1(4)$ & $1.0 \pm 0.0(3)$ & $1.0 \pm 0.0$ & $2.1 \pm 0.1(4)$ & $2.0 \pm 0.2(3)$ & $2.5 \pm 0.3(4)$ \\
\hline C22:6w3 (\%) & $6.8 \pm 0.6(4)$ & $6.2 \pm 0.4$ & $6.4 \pm 0.3$ & $7.0 \pm 1.1(4)$ & $7.1 \pm 1.7(3)$ & $7.8 \pm 1.3(4)$ \\
\hline
\end{tabular}

N.D., not detected.

In both STZ and GK rats, the injection of the MCT:FO emulsion increased the ponderal percentage of $\mathrm{C} 20: 5 \mathrm{\omega} 3 \mathrm{in}$ liver phospholipids and triglycerides, the values recorded in the animals injected with the MCT:FO emulsion averaging $139.0 \pm 12.1 \%(n=16 ; \mathrm{p}<0.01)$ of the mean corresponding values found in the same type of diabetic rats (STZ or GK rats) and in the same liver lipids (phospholipids or triglycerides) in animals injected either with saline or the control lipid emulsion $(100.0 \pm 7.5 \% ; n=34)$. Likewise, the injection of the MCT:FO emulsion increased significantly $(\mathrm{p}<0.05)$, in the liver triglycerides of STZ and GK rats, the ponderal percentage of $\mathrm{C} 22: 5 \omega 3$ to $128.6 \pm 13.7 \%(\mathrm{n}=8)$ of the mean corresponding control values $(100.0 \pm 6.2 \%$; $\mathrm{n}=17)$.

In the plasma triglycerides of GK rats injected with the MCT:FO emulsion, the mean values for either the absolute amount or ponderal percentage of C20:5 $23, \mathrm{C} 22: 5 \omega 3$ and C22:6w3 were always higher than in the GK rats injected with saline or the MCT:LCT control emulsion (Table II). Such a difference achieved statistical significance $(\mathrm{p}<0.05)$ in the case of the absolute amount of C20:5 $13(6.82 \pm 0.97 \mu \mathrm{g} / \mathrm{ml}$; $\mathrm{n}=4$ versus $4.44 \pm 0.43 \mu \mathrm{g} / \mathrm{ml} ; \mathrm{n}=7)$ and its ponderal percentage $(1.58 \pm 0.12 \% ; n=4$ versus $1.30 \pm 0.06 \% ; n=7)$. The ponderal percentage of $\mathrm{C} 22: 503$ in the plasma triglycerides was almost significantly higher $(\mathrm{p}<0.06)$ in the GK rats injected with the MCT:FO emulsion $(2.46 \pm 0.25 \% ; n=4)$ than in the other GK rats $(2.00 \pm 0.07 \% ; \mathrm{n}=7)$.

Another change in the liver phospholipid caused by the injection of the MCT:FO emulsion consisted in an increase of the paired $\mathrm{C} 18: 2 \omega 6 / \mathrm{C} 18: 3 \omega 6$ ratio for the ponderal percentage of these two fatty acids. Thus, relative to the reference value found in animals of the same type (STZ or GK rats) injected with saline $(100.0 \pm 4.4 \% ; n=9)$, such a paired ratio averaged $107.7 \pm 8.2 \%(n=8 ; p>0.4)$ and $127.1 \pm 12.2 \%(n=8 ; p<0.05)$ in the rats injected with the control lipid emulsion and MCT:FO emulsion, respectively. The latter response to the MCT:FO emulsion represents a mirror image of the situation found when comparing $\omega 3$-depleted rats (second generation) to normal animals.

These changes coincided with a decrease in the ponderal percentage of selected fatty acids in the liver triglycerides. For instance, in the STZ rats, the ponderal percentage of C18:3 13 and $\mathrm{C} 20: 4 \omega 6$ was decreased $(\mathrm{p}<0.05)$, in the animals injected with the MCT:FO emulsion to $81.9 \pm 9.2 \%(n=8)$ of the mean corresponding values found for the same fatty acid in STZ rats injected with either saline or the control lipid emulsion $(100.0 \pm 3.9 \% ; n=20)$. A comparable situation was observed in the liver triglycerides of GK rats in the case of C20:4 $\omega 6$ and C22:4w6. Pooling together the results of these two series of observations, the results recorded in the diabetic rats injected with the MCT:FO emulsion averaged $86.0 \pm 6.0 \% \quad(n=16$; $\mathrm{p}<0.06$ ) of the mean corresponding values found in the other animals $(100.0 \pm 4.0 \% ; n=34)$. For the purpose of comparison, the ponderal percentage of $\mathrm{C} 16: 0$ in the liver triglycerides averaged, in the rats injected with saline or the MCT:LCT emulsion and those injected with the MCT:FO emulsion, respectively, $22.7 \pm 0.9 \%(\mathrm{n}=10)$ and $22.3 \pm 1.7 \%(\mathrm{n}=4)$ in STZ rats, and $24.6 \pm 0.7 \%(n=7)$ and $24.3 \pm 0.9 \%(n=4)$ in GK rats.

At this point, it should be underlined that, at variance with the situation previously documented in $\omega 3$-depleted rats (second generation), the injection of the MCT:FO emulsion failed to affect significantly ( $>>0.45)$, the total fatty acid content of liver triglycerides.

The results summarized in Table I also reveal significant differences between the two animal models of diabetes examined in this study. First, and as already mentioned, the fatty acids that displayed a decreased ponderal percentage in liver triglycerides after injection of the MCT:FO emulsion were not identical in STZ and GK rats. Second, differences between these two types of rats were observed in the composition of liver phospholipids. For instance, the ponderal 


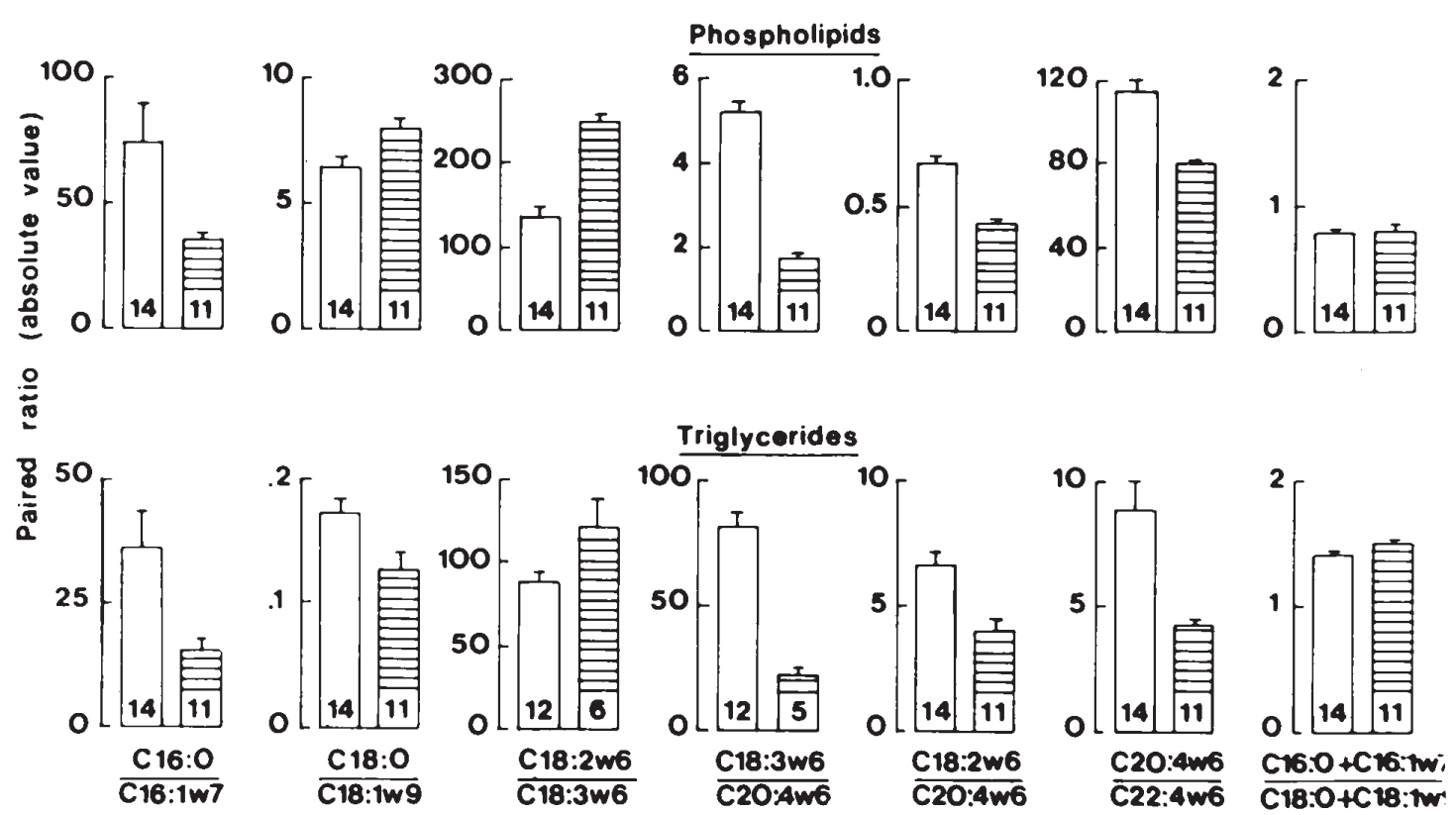

Figure 1. Paired ratio between the ponderal amount of selected fatty acids in the liver phospholipids (upper panel) and triglycerides (lower panel) of STZ rats (open columns) and GK (hatched columns). Mean values ( \pm SEM) refer to the number of individual determinations indicated at the bottom of each column. The paired $\mathrm{C} 18: 3 \omega 6 / \mathrm{C} 20: 4 \omega 6$ ratios were multiplied by $10^{3}$.

percentage of both $\mathrm{C} 20: 4 \omega 6$ and $\mathrm{C} 22: 6 \omega 3$ in hepatic phospholipids were higher $(\mathrm{p}<0.001)$ in the GK rats than in the STZ rats with mean respective values of $33.5 \pm 0.5 \%$ $(n=11)$ and $29.9 \pm 0.6 \%(n=14)$ in the case of $C 20: 4 \omega 6$ and $9.7 \pm 0.2 \%(n=11)$ and $6.4 \pm 0.3 \%(n=14)$ in the case of $C 22: 6 \omega 3$. Inversely, the ponderal percentage of $\mathrm{C} 18: 2 \omega 6$ in the liver phospholipids was higher $(\mathrm{p}<0.001)$ in STZ rats $(19.9 \pm 0.4 \%$; $\mathrm{n}=14)$ than in GK rats $(14.2 \pm 0.3 \% ; \mathrm{n}=11)$. Third, the total liver triglyceride content, expressed as fatty acid equivalent, was much higher $(\mathrm{p}<0.006)$ in GK rats $(9.08 \pm 1.84 \mathrm{mg} / \mathrm{g}$ wet weight; $\mathrm{n}=11)$ than in STZ rats $(3.73 \pm 0.32 \mathrm{mg} / \mathrm{g} ; \mathrm{n}=13)$. Last, the ponderal percentage of several fatty acids in the liver triglycerides also differed in the two animal models of diabetes. For instance, the ponderal percentage of $\mathrm{C} 22: 5 \omega 3, \mathrm{C} 22: 6 \omega 3$ and $C 20: 4 \omega 6$ were all significantly higher $(\mathrm{p}<0.03)$ in the triglycerides of GK rats than in those of STZ rats. Once again, the inverse situation prevailed in the case of C18:2 $\omega 6$ $(\mathrm{p}<0.001)$.

\section{Discussion}

The present results reveal that, even $20 \mathrm{~h}$ after the intravenous bolus injection of the MCT:FO emulsion, the content of liver phospholipids and triglycerides in long-chain polyunsaturated $\omega 3$ fatty acids remains significantly higher than in control animals injected with either saline or the MCT:LCT emulsion. Moreover, they document that these changes coincide with a change in the $\mathrm{C} 18: 2 \omega 6 / \mathrm{C} 18: 3 \omega 6$ paired ratio in the liver phospholipids in a mirror image of that otherwise caused by a depletion of long-chain polyunsaturated $\omega 3$-fatty acids. They also coincide with a decrease in the triglyceride content of

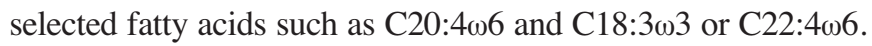
The latter situation is reminiscent of that found in $\omega 3$-depleted rats examined $60 \mathrm{~min}$ after the bolus injection of the MCT:FO emulsion, in which case even the total triglyceride content of the liver was decreased. In both the latter and present experiments, this metabolic response may well be attributable to the enrichment of membrane phospholipids in longchain polyunsaturated $\omega 3$ fatty acids and/or changes in the expression of genes involved in fatty acid synthesis and oxidation (6).

The regulatory action of the MCT:FO emulsion on liver metabolism does not represent the sole potential benefit of the present procedure. For instance, it was documented in the same STZ rats as those used in this study, that the MCT:FO emulsion protects aortic endothelial function against the deleterious effect of oxidized low density lipoproteins (Fontaine D, et al, J Parenter Enteral Nutr 29: abs. S26, 2005).

Another interesting finding emerging from the present measurements concerns the difference between STZ and GK rats in terms of both the total hepatic triglyceride content and the composition of liver phospholipids and triglycerides in selected fatty acids. The much higher triglyceride content of the liver in GK rats as compared to STZ rats coincides with a much higher insulinemia in the former as compared to latter diabetic animals. The greatest difference in the composition of

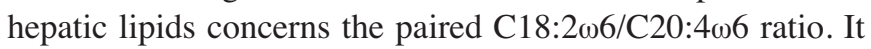
averaged, in the phospholipids and triglycerides respectively, $0.672 \pm 0.026$ and $6.669 \pm 0.518(\mathrm{n}=14$ in both cases $)$ in STZ rats, as distinct $(\mathrm{p}<0.002)$ from $0.431 \pm 0.016$ and $4.035 \pm 0.435$ $(\mathrm{n}=11$ in both cases) in GK rats. This suggests a higher activity in $\Delta 6$ - and/or $\Delta 5$-desaturase(s) in the GK rats than in the STZ rats. In order to distinguish between the latter two desaturases, the paired $\mathrm{C} 18: 2 \omega 6 / \mathrm{C} 18: 3 \omega 6$ and $\mathrm{C} 18: 3 \omega 6 / \mathrm{C} 20: 4 \omega 6$ ratios were established in the phospholipids and triglycerides of both STZ and GK rats. As illustrated in Fig. 1, in both the liver phospholipids and triglycerides, the $\mathrm{C} 18: 2 \omega 6 / \mathrm{C} 18: 3 \omega 6$ paired ratios were higher $(\mathrm{p}<0.05)$ in the GK than STZ rats, whilst the opposite situation prevailed $(\mathrm{p}<0.001)$ in the case of the

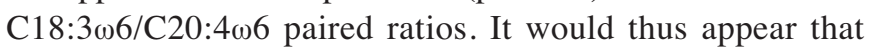




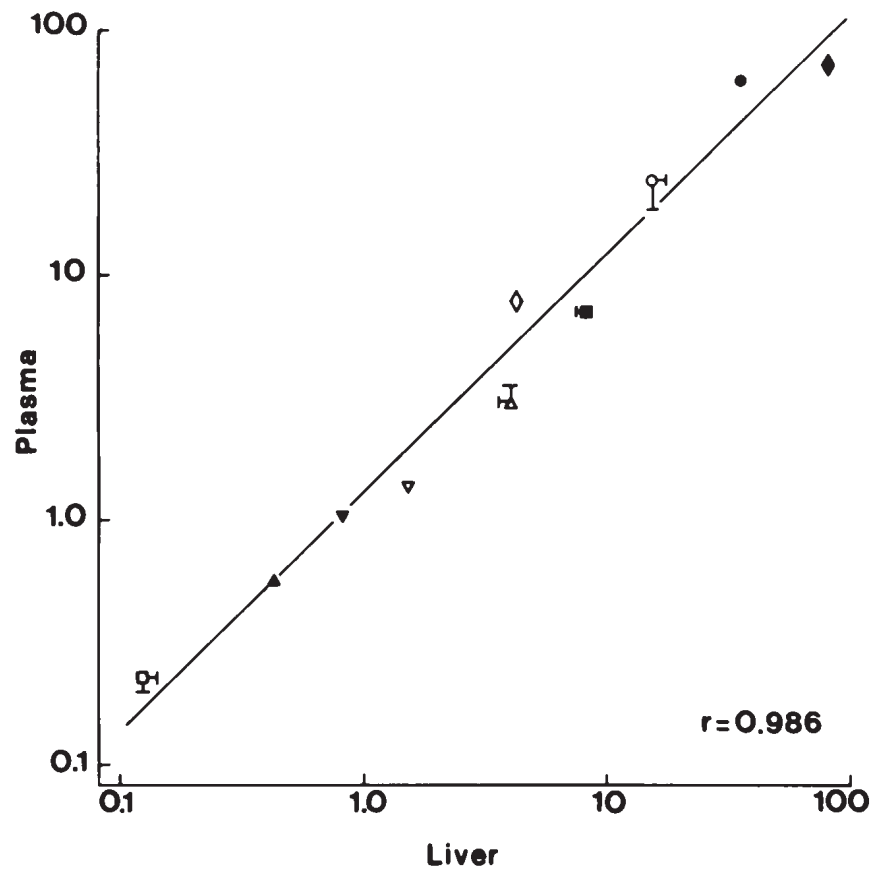

Figure 2. Correlation between the plasma and liver values for the $\mathrm{C} 16: 0$ /

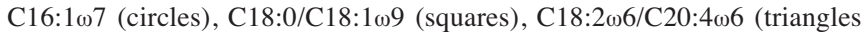
pointing upwards), $(\mathrm{C} 16: 0+\mathrm{C} 16: 1 \omega 7) /(\mathrm{C} 18: 0+\mathrm{C} 18: 1 \omega 9)$ (triangles pointing downwards) and $\mathrm{C} 20: 4 \omega 6 / \mathrm{C} 22: 4 \omega 6$ (diamonds) paired ratios in the phospholipids (closed symbols) and triglycerides (open symbols) of GK rats. Mean values ( \pm SEM, whenever exceeding the size of the mean point) refer to 10-11 individual determinations, are ranged on a logarithmic scale, and are shown together with the correlation coefficient (r).

either an elongase or the $\Delta 5$-desaturase represents the key enzyme regulating the stepwise conversion of C18:2 $\omega 6$ to C20:4w6. The possible participation of the elongase is compatible with the findings relative to the $C 20: 4 \omega 6 / C 22: 4 \omega 6$ paired ratios in both the phospholipids and triglycerides of STZ versus GK rats (Fig. 1). However, no significant difference $(p>0.09)$ was observed when comparing the $(\mathrm{C} 16: 0+\mathrm{C} 16: 1 \omega 7) /(\mathrm{C} 18: 0+\mathrm{C} 18: 1 \omega 9)$ paired ratio in either the liver phospholipids or triglycerides of STZ versus GK rats (Fig. 1). The activity of $\Delta 9$-desaturase as judged from the paired $\mathrm{C} 16: 0 / \mathrm{C} 16: 1 \omega 7$ ratio in ponderal percentage was twice higher in the liver of GK rats than STZ rats, whether in phospholipids $(74.2 \pm 15.3 ; \mathrm{n}=14$ in STZ rats versus 35.6 \pm 2.3 ; $\mathrm{n}=11$ in $\mathrm{GK}$ rats; $\mathrm{p}<0.05)$ or triglycerides $(35.9 \pm 7.4 ; \mathrm{n}=14$ in STZ rats versus $15.5 \pm 2.2 ; n=11$ in GK rats; $\mathrm{p}<0.05)$, the ratio found in GK rats averaging in these two lipid classes $45.6 \pm 3.4 \%(n=22 ; p<0.005)$ of the mean corresponding value found in STZ rats $(100.0 \pm 14.3 \% ; n=28)$. In the case of the C18:0/C18:1 $\omega 9$ paired ratio in ponderal percentage, the value found in the triglycerides of STZ rats $(0.173 \pm 0.012 ; n=14)$ was also significantly higher $(\mathrm{p}<0.01)$ than that found in the triglycerides of GK rats $(0.126 \pm 0.016 ; n=11)$, again suggesting a higher activity of the $\Delta 9$-desaturase in GK rats than in STZ rats. However, an opposite situation was found at the level of the hepatic phospholipids, the paired $\mathrm{C} 18: 0 / \mathrm{C} 18: 1 \omega 9$ ratio being now higher $(\mathrm{p}<0.02)$ in GK rats $(8.00 \pm 0.44 ; \mathrm{n}=11)$ than in STZ rats $(6.46 \pm 0.39 ; n=14)$.

Fig. 2 illustrates the close correlation $(r=0.986 ; p<0.001)$ between the liver and plasma mean values for the C16:0/

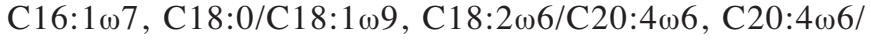
$\mathrm{C} 22: 4 \omega 6$ and $(\mathrm{C} 16: 0+\mathrm{C} 16: 1 \omega 7) /(\mathrm{C} 18: 0+\mathrm{C} 18: 1 \omega 9)$ paired ratios found in both the phospholipids and triglycerides of GK rats. The slope of the regression line $(0.9612 \pm 0.0576)$ was not significantly different $(\mathrm{p}>0.5)$ from that of a line defining identity between the two sets of values. Since all of these ratios were recently found to be affected in rats depleted in longchain polyunsaturated $\omega 3$-fatty acids (second generation), their measurements in selected plasma lipids could conceivably represent an approach to assess the metabolic consequences of such a depletion in human subjects and their possible correction by a suitable dietary management. This approach would then present the further advantage to document such a depletion in circulating lipids in which the amount of C20:5 23 may be normally too low to allow its detection, as was indeed the case in the present study for the plasma phospholipids of GK rats.

In conclusion, the present study documents in animal models of both Type 1 and Type 2 diabetes a sustained enrichment of liver lipids and plasma triglycerides in longchain polyunsaturated $\omega 3$ fatty acids and a concomitant remodelling of triglyceride composition in response to the bolus intravenous injection of the MCT:FO emulsion. In normal human subjects, it was already documented that such an injection increases within $60 \mathrm{~min}$ and for 24-48 h the phospholipid content in C20:5 133 and $C 22: 6 \omega 3$ of circulating platelets and leucocytes. In light of the present findings, it may be expected, therefore, that the potential beneficial metabolic and functional effects of such an enrichment in other cell types (e.g. hepatocytes and vascular endothelial cells) will follow a comparable time course.

\section{Acknowledgements}

This study was supported by a grant from the Région de Bruxelles-Capitale (Convention BC-BR 246/2410, Brussels, Belgium) to Target Hit s.a. (Brussels, Belgium). The lipid emulsions were kindly provided by B. Braun Melsungen (Melsungen, Germany). We are grateful to A. Chwalik, P. D'Hont and A. Dufour for their technical assistance and C. Demesmaeker for secretarial help.

\section{References}

1. Richelle M, Carpentier YA and Deckelbaum RJ: Long- and medium-chain triacylglycerols in neutral lipid-exchange processes with human plasma low-density lipoproteins. Biochemistry 33: 4872-4878, 1984.

2. Malaisse WJ, Ladrière L, Kadiata MM and Malaisse-Lagae F: Pancreatic fate of ${ }^{14} \mathrm{C}$-labelled hexoses. Cell Biochem Funct 18: 281-291, 2000.

3. Malaisse WJ, Ladrière L and Malaisse-Lagae F: Pancreatic fate of 6-deoxy-6-[ $\left.{ }^{125} \mathrm{I}\right]$ iodo-D-glucose. In vivo experiments. Endocrine 13: 95-101, 2000.

4. Giroix M-H, Scruel O, Ladrière L, Sener A, Portha B and Malaisse WJ: Metabolic and secretory interactions between D-glucose and D-fructose in islets from GK rats. Endocrinology 140: 5556-5565, 1999.

5. Laghmich A, Ladrière L, Malaisse-Lagae F and Malaisse WJ: Long-term effects of glibenclamide and nateglinide upon pancreatic islet function in normal and diabetic rats. Pharmacol Res 40: 475-482, 1999.

6. Benatti P, Peluso G, Nicolai R and Caluani M: Polyunsaturated fatty acids : biochemical, nutritional and epigenetic properties. J Am Coll Nutr 23: 281-302, 2004. 